\title{
DIAGNÓSTICO SITUCIONAL EM UNIDADE DE TERAPIA INTENSIVA: RELATO DE EXPERIÊNCIA ${ }^{1}$
}

\author{
SITUATIONAL DIAGNOSTIC IN AN INTENSIVE CARE UNIT: REPORT OF AN \\ EXPERIENCE
}

\section{DIAGNÓSTICO SITUCIONAL EN LA UNIDAD DE CUIDADOS INTENSIVOS: INFORME DE LA EXPERIENCIA}

\author{
BIANCA MARIA OLIVEIRA LUVISARO \\ Instituto Nacional de Câncer-INCA/RJ \\ biancaluvisaro@hotmail.com
}

GABRIELLA SANTOS LIMA
Universidade de São Paulo-USP
gabriellasantos_3@ @otmail.com

\author{
ELANA MARIA RAMOS FREIRE \\ Universidade Federal de Minas Gerais-UFMG \\ elanafreire@gmail.com
}

\author{
MARIA REGINA MARTINEZ \\ Universidade Federal de Alfenasl-Unifal-MG \\ martinez@unifal-mg.edu.br
}

\begin{abstract}
RESUMO
O presente trabalho trata-se de um relato de experiência que teve como objetivo realizar um diagnóstico situacional de uma Unidade de Terapia Intensiva (UTI) de um hospital privado de pequeno porte, visando identificar não conformidades e desenvolver estratégias de melhoria para este setor. Os dados foram coletados por meio de uma lista de verificação simples e mapeamento de processos, a fim de conhecer a realidade do serviço e identificar problemas.Após análise dos resultados foram listadas as não conformidades e elaborado propostas de melhoria dos serviços prestados. A partir da análise dos dados e do mapeamento de processos, pode-se identificar problemas na UTI, destacando-se dentre eles fatores relacionados à ausência de um enfermeiro específico no setor. Desta forma foi proposta a implantação da Sistematização da Assistência de Enfermagem (SAE), uma vez que facilitaria a padronização da assistência dos técnicos de enfermagem, auxiliando na monitorização e na maior participação de enfermeiros na unidade.
\end{abstract}

PALAVRAS-CHAVE: Gestão de qualidade, UTI, Enfermagem.

\begin{abstract}
The objective of the present paper is a situational diagnostical analysis in an (ICU), at a small private hospital in order to identify non-compliance and develop strategical improvement for this sector. Data were collected and mapped through a simple checklist, to determine problems actually rendered services. After the analyzing the results, the non-compliance factors have been listed and proposals were elabored to improve the services. Based on the dada results, problems in the ICU were identified, standing out the absence of a nurse in the unit. Therefore, the implementation of Nursing Care System (NCS) has been proposed since it would facilitate the standardization of the nurse technicians, ensuring better monitoring and participation by all the member of the nursing staff in the unit.
\end{abstract}

Keywords: Quality management, ICU, Nursing.

\section{RESUMEN}

El presente trabajo es un relato de experiencia que tuvo como objetivo realizar un diagnóstico situacional de la Unidad de Cuidados Intensivos (UCI) de un pequeño hospital privado, para identificar no conformidades y desarrollar estrategias de mejora para este sector. Los datos fueron recolectados a través de una lista de control simple y mapeo de procesos con el fin de conocer la realidad del servicio e identificar problemas.Após resultados de los análisis se enumeran las no conformidades y elaborar propuestas para la mejora de los servicios. A partir del análisis de datos y el proceso de asignación, puede identificar problemas en la UCI, especialmente entre los

\footnotetext{
${ }^{1}$ Submetido em 03 de maio de 2013. Aceito em 12 de outubro de 2013. O artigo foi avaliado segundo o processo de duplo anonimato e pelos editores. Editores responsáveis: Márcio Augusto Gonçalves e Lucas Maia dos Santos. Reprodução parcial ou total e trabalhos derivativos permitidos com a citação apropriada da fonte.
} 

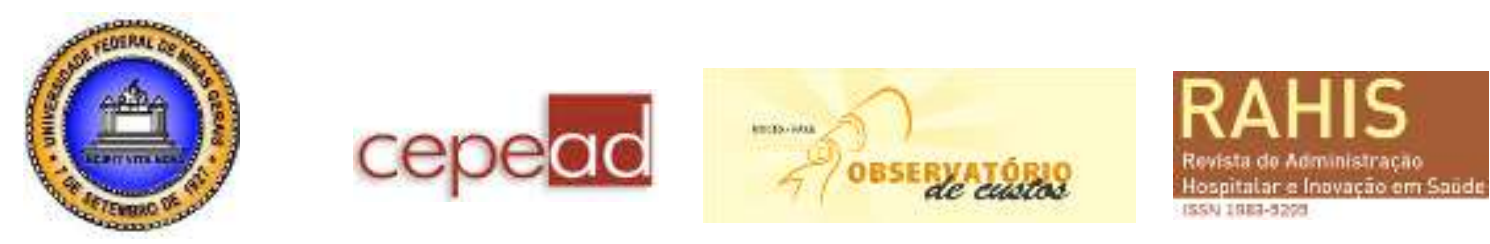

factores relacionados con la falta de enfermeras en un sector específico. Por lo tanto, se propuso la implementación de la Sistematización de Enfermería (SAE), ya que facilitaría la normalización de la atención de los técnicos de enfermería, la asistencia en el seguimiento y la mayor participación de las enfermeras en la unidad. PALABRAS CLAVE: Gestión de la calidad, la UCI, Enfermería.

\section{INTRODUÇÃO}

A melhora da qualidade na prestação dos serviços hospitalares depende da reorganização estrutural e do remodelamento dos processos de atendimento, além do desenvolvimento de uma cultura organizacional voltada para a Gestão de Qualidade (MALIK; TELES, 2001; PERTENCE; MELLEIRO, 2010).

A melhoria contínua da qualidade hospitalar tem sido buscada mais intensamente pelos hospitais como um fator de atratividade e fidelização de clientes, favorecendo sua manutenção no mercado de prestação de serviços de saúde, setor que tem se tornado cada vez mais competitivo com a reestruturação da atenção primária no país e envelhecimento da população (SOUZA; COSTA, 2011).

No Brasil, a melhoria da qualidade hospitalar tem sido impulsionada por selos de certificação que atestam os esforços organizacionais no sentido de oferecer serviços de excelência. Essa certificação é fornecida pela Organização Nacional de Acreditação Hospitalar (ONA, 2010).

A ONA é uma organização privada, sem fins lucrativos e de interesse coletivo, que tem como principais objetivos a implantação e implementação nacional de um processo permanente de melhoria da qualidade da assistência à saúde, estimulando todos os serviços de saúde a atingirem padrões mais elevados de qualidade, dentro do Processo de Acreditação (FELDMAN et al, 2005).

A Acreditação Hospitalar é um procedimento de avaliação dos recursos institucionais, voluntário, periódico, reservado e sigiloso, que tende a garantir a qualidade da assistência através de padrões previamente aceitos. Os padrões podem ser mínimos ou mais elaborados e exigentes, definindo diferentes níveis de satisfação e qualificação, sendo os mesmos: nível I ou Acreditado, nível II ou Acreditado Pleno e nível III ou Acreditado com Excelência(ONA, 2010).

No nível I os requisitos básicos são voltados para estrutura física e segurança, visando garantir uma assistência orientada para a execução coerente de suas tarefas. O nível II engloba a existência de normas e rotinas e procedimentos documentados e aplicados, utilizando uma lógica de melhoria dos processos de assistência e nos procedimentos médico-sanitários. Para alcançar a excelência em gestão,nível III, as instituições devem possuir políticas institucionais de melhoria contínua em termos de estrutura, novas tecnologias, atualização técnicoprofissional, ações assistenciais e monitoramento de procedimentos médico-sanitários (ALVES, 2009).

Para se atingir os níveis de qualificação e satisfação de qualidade previstos pela ONA, os hospitais têm se profissionalizado na utilização de ferramentas gerencias de qualidade hospitalar. Dentre as ferramentas disponíveis, o diagnóstico situacional torna-se uma importante ferramenta de gestão uma vez que proporciona uma análise do ambiente organizacional contribuindo para identificar a real situação de um setor bem como os problemas, necessidades e oportunidades deste.

Este trabalho descreve o diagnóstico situacional de uma Unidade de Tratamento Intensivo (UTI) hospitalar, onde essa ferramenta gerencial foi utilizada como uma estratégia de 

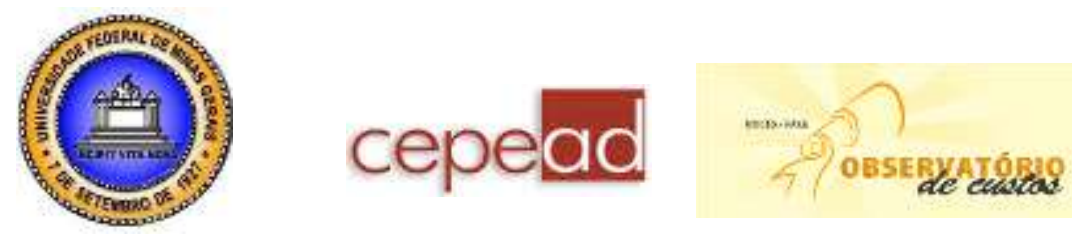

identificação de problemas e desenvolvimento de soluções durante o processo de ensinoaprendizagem de alunos de graduação de enfermagem em atividades práticas de gerenciamento de enfermagem. O diagnóstico situacional trata-se do levantamento de uma situação real de uma organização, com intuito de conhecer a empresa ou o negócio, sendo uma das mais importantes ferramentas de gestão (BAULI, MATSUDA, 2009).

De acordo com o Ministério da Saúde, a UTI é um local de grande especialização e tecnologia, identificado como espaço laboral destinado a profissionais da saúde, principalmente médicos e enfermeiros, possuidores de grande aporte de conhecimento, habilidades e destreza para a realização de procedimentos. Nesse sentido, subentende-se que, os profissionais que atuam nessas unidades, necessitam de muito preparo, pois invariavelmente, podem se defrontar com situações cujas decisões definem o limite entre a vida ou a morte das pessoas (INOUE, 2009).

Por se tratar de um setor de alta complexidade tecnológica e de procedimentos, a UTI deve ter seus processos bem estruturados e mapeados, a fim de orientar a tomada de decisões dos profissionais que ali atuam facilitando o entendimento do fluxo do setor.

Princípios de gerenciamento que utilizam o mapeamento e remodelamento de processos auxiliam as organizações hospitalares a reverem seus fluxos de atendimento nos diversos setores do hospital e a buscarem alternativas que resultem em redução de custo, diminuição do desperdício de tempo e materiais, aumento da eficiência e aumento da satisfação dos seus colaboradores e clientes (COLLIGAN et al., 2010; JOHNSON, BARACH, 2011; RAVEN et al, 2011).

Neste trabalho, a partir da utilização de ferramentas de qualidade, foram identificadas em uma UTI não conformidades referentes à área física, a recursos humanos e materiais. Em seguida, após a análise das não conformidades, apresentam-se instrumentos estratégicos visando atender às adequações necessárias para melhoria qualidade da assistência no setor.

\section{MÉTODO}

O presente trabalho trata-se de um estudo descritivo, do tipo relato de experiência, cujo objetivo foi realizar um diagnóstico situacional de uma UTI de um hospital privado de pequeno porte de um município do Sul de Minas Gerais, visando identificar não conformidades e desenvolver estratégias de melhoria para este setor.

Os dados foram coletados por meio de uma lista de verificação simples (GRUPO TÉCNICO DE ACREDITAÇÃO DE HOSPITAIS, 1997)e pelo mapeamento de processos do setor.

A lista de verificação simples é utilizada para coletar informações de itens préestabelecidos que precisam ser verificados, possibilitando uma melhor percepção da realidade e uma imediata interpretação da situação. É uma ferramenta que facilita o entendimento do problema, pois coletar os dados de forma orientada evita decisões por opiniões e as tornam embasadas em fatos reais (ALVES, 2012).

O mapeamento de processos é um diagrama mostrando a sequência de passos e decisões utilizadas para acompanhar um procedimento do seu início até seu fim. É uma ferramenta que não costuma ser utilizada rotineiramente nas organizações de saúde, mas tem sido reconhecida como valiosa para a reestruturação dos serviços e melhoria da qualidade hospitalar (ANJARD, 1996; FENTON, 2007; RATH, 2008).

Após a análise dos resultados foram listadas as não conformidades identificadas, cada não conformidade foi justificada por meio de estudos de normas e legislação vigente, em 
seguida foram propostas estratégias de melhoria da qualidade e estruturação do serviço, considerando a realidade da organização em estudo.

\section{RESULTADOS E DISCUSSÃO}

\section{AMBIENTE DA UNIDADE DE TERAPIA INTENSIVA}

A Unidade de Terapia Intensiva em estudo é composta por cinco leitos sendo um de isolamento, com taxa de ocupação variável. O quadro profissional é constituído por três profissionais de enfermagem por plantão, sendo dois técnicos e um enfermeiro. O fluxo de trabalho da UTI em questão é dividido em três fases: admissão, internação e alta. A admissão é realizada por meio de uma avaliação médica de pacientes oriundos de setores internos (pronto atendimento, bloco cirúrgico, unidade de internação) ou externos (transferência hospitalar). Após avaliação médica os pacientes admitidos na UTI passam por uma rotina diária de exames, procedimentos, avaliação médica e de enfermagem até receberam alta do setor, conforme pode ser visto no mapeamento de processos (FIGURA 1). 


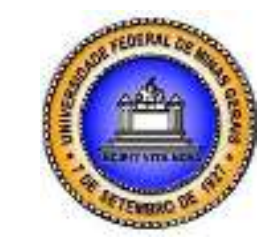

\section{cepead}

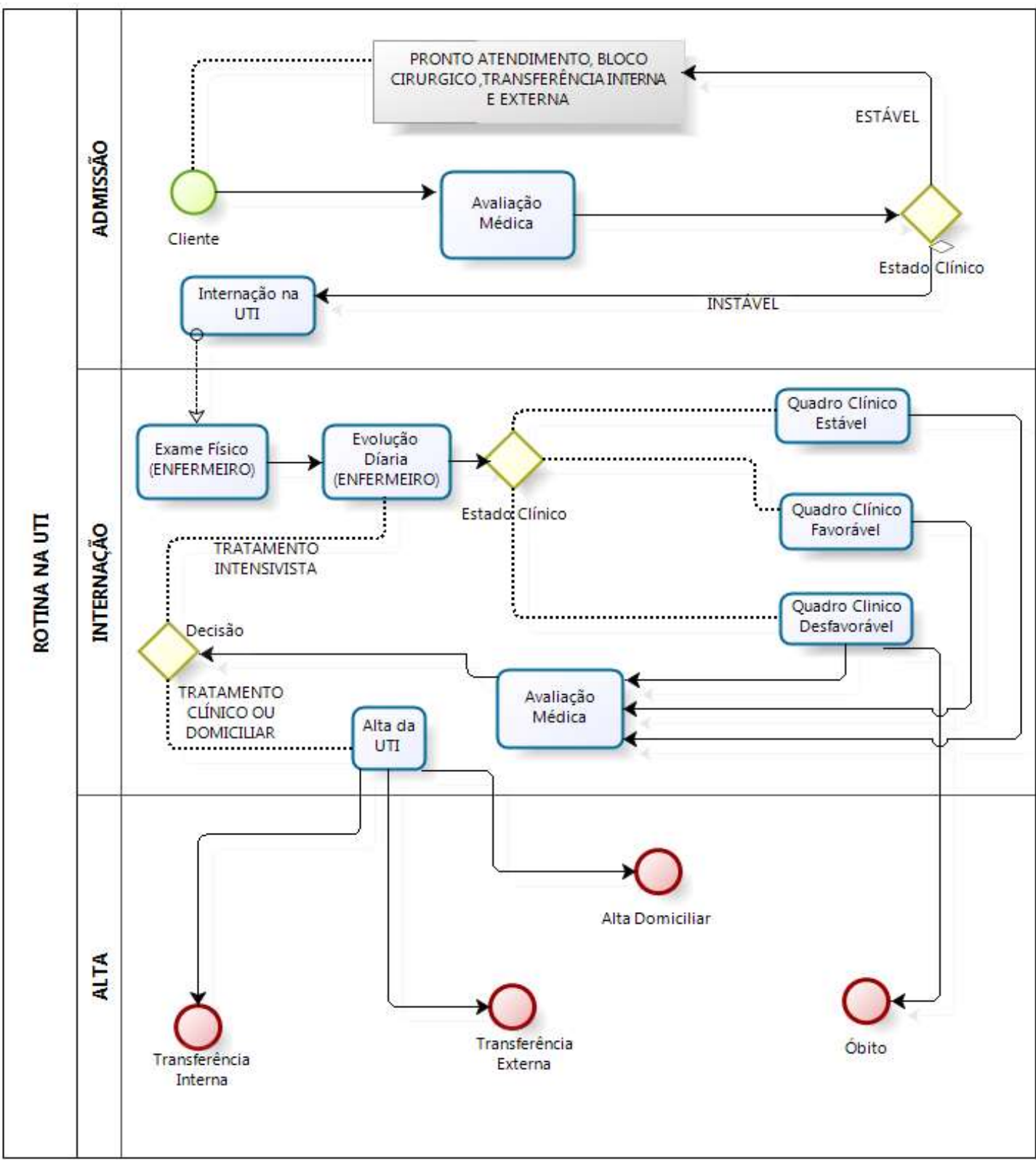

Figura 1 - Mapeamento dos processos da UTI de um hospital privado de pequeno porte, Sul de Minas Gerais, 2012.

O mapeamento de processos possibilitou a visualização de pontos fortes e fracos no atendimento da UTI, traçando o fluxo dos serviços de enfermagem nesta unidade possibilitando identificar necessidades de melhoria durante a rotina de admissão, internação e alta do cliente, cumprindo o seu papel de ferramenta gerencial e de comunicação. Essa ferramenta busca descrever graficamente um processo novo ou existente, usando símbolos, linhas e texto, para representar as atividades, sequência dos processos e o agente executor (MELLO; SALGADO, 2005). 
O mapeamento de processos é mais que um simples instrumento de visualização da prestação de um serviço hospitalar, pois pode levar a um entendimento amplo de como a organização trabalha, propiciando o fornecimento de dados capazes de sustentar os processos decisórios e o planejamento estratégico organizacional (VELILLA; MARTÍNEZ, 2012).

\section{NÃO CONFORMIDADES IDENTIFICADAS}

Após análise da lista de verificação e do mapeamento dos processos foi possível levantar as não conformidades da UTI, que seguem listadas no Quadro 1. Cada não conformidade identificada foi justificada por meio de normas e legislação vigente e foram correlacionadas com a realidade encontrada, somando-se ainda a justificativa pela coordenação da UTI e Serviço de Controle de Infecção Hospitalar (SCIH) sobre o não cumprimento do requisito.

\section{TABELA 1- Não conformidades identificadas na UTI de um hospital privado de pequeno porte, Sul de Minas Gerais, 2012.}

\begin{tabular}{|l|l|l|}
\hline Requisitos exigidos em uma Unidade de Terapia Intensiva & SIM & NÃO \\
\hline 1- Há médico exclusivo responsável pela unidade 24 horas por dia? & & $\mathrm{X}$ \\
\hline 2- Há enfermeiro exclusivo da unidade? & & $\mathrm{X}$ \\
\hline 3- Há disponível e exclusivo oftalmoscópio na unidade? & & $\mathrm{X}$ \\
\hline 4- Há médico intensivista disponível durante 24 horas? & $\mathrm{X}$ \\
\hline $\begin{array}{l}\text { 5- Há enfermeiro capacitado em atendimento de UTI exclusivo da unidade durante } \\
\text { 24 horas? }\end{array}$ & & $\mathrm{X}$ \\
\hline 6- Todos os médicos e plantonistas têm especialização em medicina intensiva? & & $\mathrm{X}$ \\
\hline 7- Todo o pessoal de enfermagem é especializado em terapia intensiva? & & $\mathrm{X}$ \\
\hline 8- Existe capinógrafo disponível para a unidade? & $\mathrm{X}$ \\
\hline
\end{tabular}

Os itens 1 e 2, ausência de médicos e enfermeiros exclusivos 24 horas na unidade encontram-se em não conformidades.De acordo com a RDC $\mathrm{N}^{\circ} 7$ de fevereiro de 2010 uma equipe de UTI deve ser composta por: médicos plantonistas, no mínimo um para cada dez leitos ou fração, em cada turno; enfermeiros assistenciais, no mínimo um para cada oito leitos ou fração, em cada turno (ANVISA, 2010). A unidade avaliada possui um enfermeiro coordenador responsável pela supervisão dos serviços e um médico do pronto-atendimento dá suporte ao acompanhamento clínico. Durante a rotina de internação da UTI o exame físico geral e a evolução diária do paciente deveriam ser realizados apenas pelo enfermeiro do setor, porém como não há um enfermeiro exclusivo para a unidade durante 24 horas, esse processo não ocorre de maneira correta.

Como previsto pela RDC N 7 de fevereiro de 2010 (ANVISA, 2010) em uma UTI deve conter no mínimo um aparelho oftalmoscópico exclusivo para o setor, no entanto na unidade estudada não há disponível oftalmoscópio exclusivo (item 3) sendo utilizado o aparelho pessoal do próprio médico.

O item 4 ausência de médicos intensivistas assistindo os pacientes por 24 horas, e o item 5ausência de enfermeiro capacitado em atendimento de UTI exclusivo da unidade durante 24 horas, constituem de não conformidades uma vez que a RDC $N^{\circ} 7$ (ANVISA, 2010) prevê que a equipe de uma UTI deve ser composta por um médico diarista/rotineiro, profissional legalmente habilitado, responsável pela garantia da continuidade do plano assistencial e pelo 

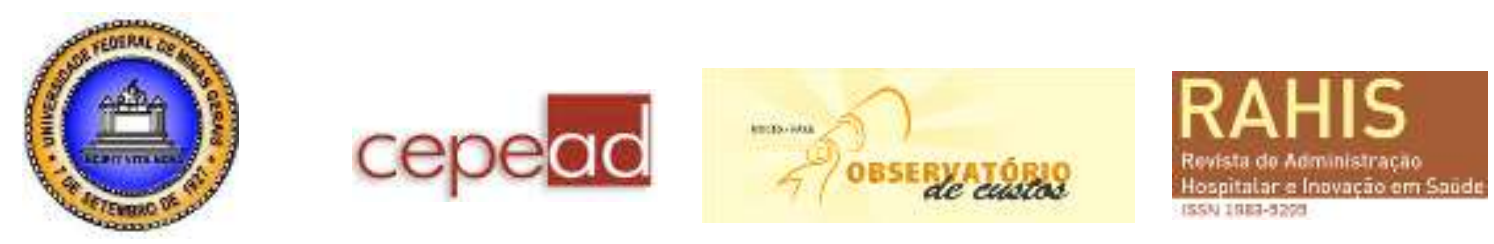

acompanhamento diário de cada paciente; médico plantonista, profissional legalmente habilitado, com atuação em regime de plantões. No entanto, na unidade em estudo apenas o médico coordenador clínico possui especialidade em UTI como indicado no item 6. Em relação à enfermagem, embora não havendo no momento um enfermeiro especialista em atendimento de UTI disponível durante 24 horas, o atual enfermeiro coordenador do setor encontra-se em treinamento e cursando especialização na área e o restante da equipe de enfermagem não possuem especialidade (item 7).

O item 8, ausência de capnógrafo disponível para unidade, constitui uma não conformidade uma vez que é previsto pela RDC $\mathrm{N}^{\circ} 7$ (ANVISA, 2010) que em uma UTI deve conter no mínimo um equipamento deste para cada 10 leitos. No setor em estudo quando necessário o uso de capnógrafo utiliza-se o aparelho disponível no Bloco Cirúrgico.

ESTRATÉGIAS A SEREM DESELVOVLVIDAS A FIM DE SANAR AS NÃO CONFORMIDADES

De acordo com as não conformidades levantadas, foi possível identificar a necessidade de implantação de um processo de Sistematização da Assistência de Enfermagem (SAE) para a UTI em questão, a fim de direcionar a assistência prestada ao paciente e aumentar a qualidade do trabalho deste setor, uma vez que não há presente 24 horas por dia um enfermeiro exclusivo para atendimento da UTI. Nesse sentido a SAE seria uma tentativa de otimizar o tempo que o enfermeiro dispõe para o setor.

A Sistematização da Assistência de Enfermagem é o modelo metodológico ideal para o enfermeiro aplicar seus conhecimentos técnico-científicos na prática assistencial, favorecendo o cuidado e a organização das condições necessárias para que ele seja realizado (BITTAR; PEREIRA; LEMOS, 2006).

Dessa forma, incorporar a SAE é uma forma de tornar a enfermagem mais científica, promovendo um cuidar de enfermagem, humanizado, contínuo, mais justo e com qualidade para o paciente/cliente.

Quando as informações estão organizadas e documentadas de forma sistematizada, a comunicação é operacionalizada e facilita a resolução dos problemas específicos de cada paciente, a fim de ampliar a visibilidade do saber da enfermagem frente ao paciente (TRUPPEL, 2009).

Neste cenário, foi proposto um plano de sistematização de enfermagem no qual foram levantados os principais diagnósticos de enfermagem em UTI (APÊNDICE A) e as principais intervenções de enfermagem para os diagnósticos listados (APÊNDICE B), baseado nos diagnósticos do NANDA (NANDA, 2010) e intervenções do NIC (MCCLOSKEY, BULECHEK, 2004) e em um levantamento bibliográfico de artigos sobre UTI, para que o enfermeiro responsável possa fazer a avaliação e deixar registradas suas prescrições diariamente, com os diagnósticos e as intervenções relevantes. Com isso os técnicos de enfermagem terão uma maior segurança em relação aos cuidados prestados e uma otimização das tarefas, contribuindo para uma padronização do serviço de enfermagem e maior qualidade no trabalho de equipe.

\section{CONCLUSÃO}

O diagnóstico situacional e o mapeamento de processos possibilitaram uma melhor visualização dos problemas na Unidade de Terapia Intensiva-UTI direcionando para implementação de estratégias de melhorias. Nota-se que o setor avaliado apresentou algumas não conformidades que dificultavam o fluxo de atendimento e assistência prestada. 

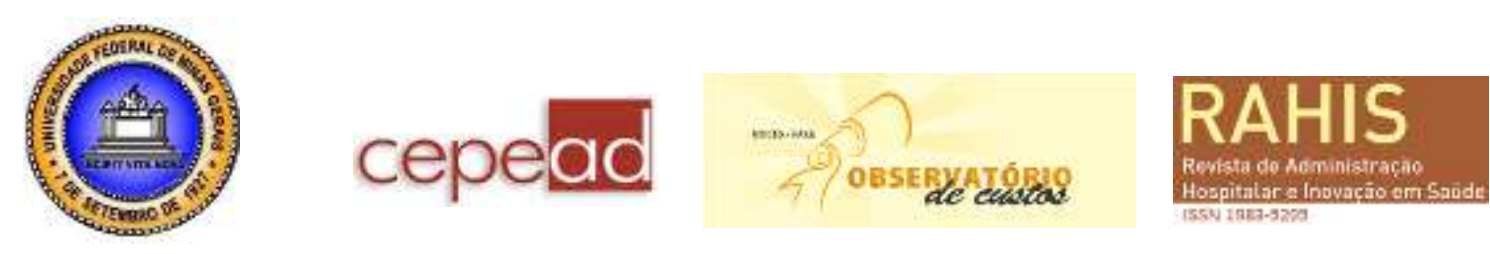

A elaboração do diagnóstico situacional acrescido do mapeamento de processos foram ferramentas chave para o desenvolvimento de estratégias de melhorias, viabilizando o desenvolvimento de um plano de assistência e cuidado, que complementam e organiza as atividades desenvolvidas pelo enfermeiro.

Com o uso do mapeamento dos processos foi possível identificar o surgimento de uma visão detalhada dos problemas e de um entendimento compartilhado do processo em estudo e o trabalho junto com SAE permite que o enfermeiro atue com maior qualidade aplicando seus princípios técnicos científicos.

Para as instituições de saúde possibilita uma melhor organização no cotidiano da equipe, pois comporta a utilização de ferramentas apropriadas que facilitam os registros, quantificação da assistência, controle de custos e auxilia a auditoria. Além de promover o alcance das metas de qualidade consegue ainda a implementação de ações, avaliação de resultados e modificação nas intervenções, favorecendo a obtenção dos resultados esperados.

\section{REFERÊNCIAS}

ALVES, V. L. S. Gestão de qualidade: ferramentas utilizadas no contexto contemporâneo da saúde. São Paulo: Martinari, 2009.

ALVES, V. L. S. Gestão da qualidade: ferramentas utilizadas no contexto contemporâneo da saúde. São Paulo: Martinari, 2.ed., 2012.

ANJARD, R.P. Process mapping: one of three, new, special quality tools for management, quality and all other professionals. Microelectron. Reliab., v. 36, n. 2, p. 223-225, 1996.

ANVISA - Agência Nacional de Vigilância Sanitária Resolução. RDC n⿳ 7, de 24 de fevereiro de 2010. Brasília, 2010.

BAULI J, D.; MATSUDA, L. M. Diagnóstico situacional do serviço de enfermagem de hospital de ensino sob a ótica dos profissionais de nível médio. Revista Administração em Saúde, v.11, n.43, p. 55-62, 2009.

BITTAR, D.B.; PEREIRA, V.P.; LEMOS, R.C.A. Sistematização da assistência de enfermagem ao paciente crítico: proposta de instrumento de coleta de dados. Texto Contexto Enfermagem, Florianópolis, v.15,n.4, p.617-628, 2006.

COLLIGAN, L., et al. Does the process map influence the outcome ofquality improvement work? A comparison of asequential flow diagram and a hierarchical taskanalysis diagram. BMC Health Services Research, v.10, n.7, 2010.

FENTON, E.M. Visualising Strategic Change: The Role and Impact of Process Maps as Boundary Objects in Reorganisation. European Management Journal, v. 25, n.2, p.104-117, 2007.

FELDMAN, L.B, et al. História da evolução da qualidade hospitalar:dos padrões a acreditação. Acta Paul Enfermagem; v.18, v.2, p.213-219, 2005. 

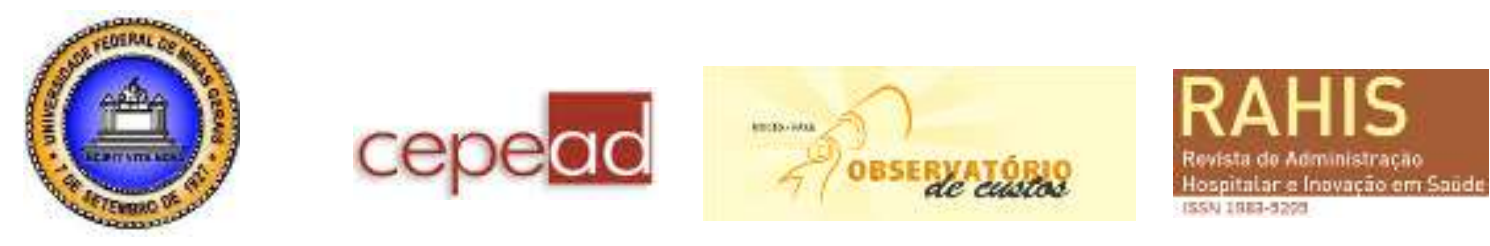

Grupo técnico de acreditação de hospitais. Manual de acreditação hospitalar. Segunda versão, 1997.

Disponível

em:

http://portalses.saude.sc.gov.br/arquivos/sala_de_leitura/saude_e_cidadania/ed_03/pdf/07_03.pdf.

Acesso em: março de 2012.

INOUE, K.C.; MATSUDA, L.M. Dimensionamento da equipe de enfermagem da UTI-adulto de um hospital ensino. Revista Eletrônica Enfermagem; v.11, n.1, p. 55-63, 2009.

JOHNSON, J.K.; BARACH, P.R. Quality improvement methods to study and improve the process and outcomes ofpediatric cardiac care. Progress in Pediatric Cardiology, v.32, p. 147-153, 2011.

MALIK, A.M.; TELES, J.P. Hospitais e programasde qualidade no estadode São Paulo. RAE Revista de Administração de Empresas, v. 41, n. 3, p.51-59, Jul./Set. 2001.

MCCLOSKEY, J.C.; BULECHEK, G.M. Classificação das intervenções de enfermagem (NIC). Porto Alegre: ARTMED; 3ed., 2004.

MELLO, C.H.P; SALGADO, E.G. Mapeamento dos processos em serviços: estudo de caso em duas pequenas empresas da área de saúde. XXV Encontro Nacional de Engenharia de Produção - Porto Alegre- RS, nov. de 2005.

NANDA. Diagnósticos de Enfermagem da NANDA: Definições e classificação 2009-2011; tradução Regina Machado Garcez - Porto Alegre: Artmed, 2010.

ONA. Manual das Organizações Prestadoras de Serviços de Saúde. Brasília: Organização Nacional de Acreditação, 2010.

PERTENCE, P.P.; MELLEIRO, M.M. Implantação de ferramenta de gestãode qualidade em Hospital Universitário. Revista Escola de Enfermagem - USP; v.44. n.4, p.1024-1031, 2010.

RATH, F.M.S.I.E. Tools for developing a quality management program: proactive tools (process mapping, value stream mapping, fault tree analysis, and failure mode and effects analysis). Int. J. Radiation Oncology Biol. Phys., v. 71, n. 1, Supplement, p. 187-190, 2008.

RAVEN, J., et al. Methodology and tools for quality improvement in maternal and newborn health care. International Journal of Gynecology and Obstetrics, v.114, p. 4-9, 2011.

SOUZA, L.L.; COSTA, J.S.D. Internações por condiçõessensíveis à atenção primária nascoordenadorias de saúde no RS. Revista Saúde Pública, n.45, v.4, p.765-772, 2011.

TRUPPEL, T.C, et al. Sistematização da Assistência de Enfermagem em Unidade de Terapia Intensiva.Revista Brasileira Enfermagem Brasília, mar-abril; v.62, n.2, p. 221-227, 2009.

VELILLAA, F.D.; MARTÍNEZ, J.R.M. Implementation of a quality model based on clinical management by processes in an orthopaedic surgery and traumatology department. RevEsp Cir Ortop Traumatol., v. 56, n.1, p. 80-93. 2012. 


\section{cepead}

\section{APÊNDICE A- DIAGNÓSTICOS DE ENFERMAGEM PARA UTI}

NOME:

LEITO:

REG.:

DATA:

\begin{tabular}{|l|l|}
\hline DIAGNÓSTICOS DE ENFERMAGEM & \\
\hline Comunicação verbal prejudicada & \\
\hline Déficit no autocuidado para banho/higiene & \\
\hline Desobstrução ineficaz de vias aéreas & \\
\hline Dor aguda & \\
\hline Risco para infecção & \\
\hline Risco para integridade da pele prejudicada & \\
\hline Risco para temperatura corporal desequilibrada & \\
\hline Ventilação espontânea prejudicada & \\
\hline Risco para aspiração & \\
\hline Perfusão tissular ineficaz & \\
\hline Nutrição desequilibrada: menos do que as necessidades corporais & \\
\hline Padrão respiratório ineficaz & \\
\hline Confusão aguda & \\
\hline Déficit no autocuidado para alimentação & \\
\hline Integridade da pele prejudicada & \\
\hline Hipertermia & \\
\hline Hipotermia & \\
\hline Mobilidade física prejudicada & \\
\hline Risco para volume de líquidos desequilibrado & \\
\hline
\end{tabular}




\section{cepead}

\section{APÊNDICE B - PRESCRIÇÕES DE ENFERMAGEM PARA UTI}

NOME:

LEITO:

REG.:

DATA:

\begin{tabular}{|c|c|}
\hline PRESCRIÇÕES DE ENFERMAGEM & \\
\hline Aspirar VAS/TOT/Traqueostomia & \\
\hline Realizar lavagem das mãos antes e após qualquer procedimento & \\
\hline Respeitar precauções e uso de EPI's conforme rotina do SCIH & \\
\hline Trocar curativos de acessos vasculares & \\
\hline Manter procedimentos datados, identificados e fixados & \\
\hline Realizar/auxiliar na higiene corporal & \\
\hline Realizar/auxiliar nas higienes oral e ocular & \\
\hline Realizar higiene íntima & \\
\hline Proporcionar mecanismos de comunicação paciente-cuidador & \\
\hline $\begin{array}{l}\text { Observar sinais de dor (taquicardia, hipertensão, taquipnéia) e comunicar ao } \\
\text { enfermeiro }\end{array}$ & \\
\hline Comunicar alterações em ventilação mecânica & \\
\hline $\begin{array}{l}\text { Observar em extremidades: cianose, perfusão, temperatura. Comunicar } \\
\text { alterações ao enfermeiro }\end{array}$ & \\
\hline Comunicar se temperatura $<35,8^{\circ} \mathrm{C}$ ou $>37,2^{\circ} \mathrm{C}$ & \\
\hline Proteger proeminências ósseas & \\
\hline Realizar mudança de decúbito a cada 2 horas & \\
\hline Hidratar e massagear pele 3 vezes ao dia & \\
\hline Realizar balanço hídrico & \\
\hline Interromper fluxo de dietas durante procedimentos & \\
\hline Aspirar SNG/SNE antes de instalar as dietas. Lavar sondas após a dieta & \\
\hline
\end{tabular}




\section{cepead}
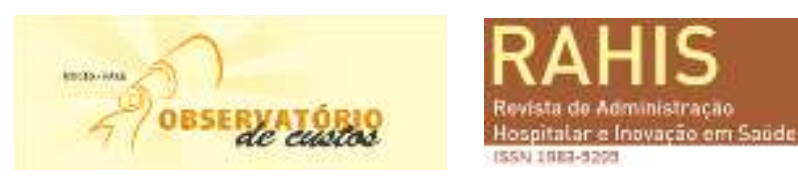

\begin{tabular}{|l|l|}
\hline Trocar acesso venoso periférico, conforme a rotina da instituição & \\
\hline Trocar umidificador/filtro conforme rotina da instituição ou SN & \\
\hline $\begin{array}{l}\text { Observar presença de edema em MMSS e MMII. Se edemaciados, elevá-los e } \\
\text { comunicar ao enfermeiro }\end{array}$ & \\
\hline Observar e anotar padrão ventilatório. Comunicar alterações ao enfermeiro & \\
\hline Comunicar ao enfermeiro se SpO2 < 92\%. & \\
\hline Instalar dietas rigorosamente nos horários & \\
\hline Conter MMSS se apresentar agitação & \\
\hline $\begin{array}{l}\text { Elevar grades laterais da cama } \\
\text { Observar e anotar freqüência de evacuações, náuseas e vômitos. Comunicar } \\
\text { alterações ao enfermeiro }\end{array}$ & \\
\hline $\begin{array}{l}\text { Observar e anotar comportamento neurológico. Comunicar alterações ao } \\
\text { enfermeiro }\end{array}$ & \\
\hline \begin{tabular}{l} 
Suspender procedimentos se PIC > 20 mmHg e comunicar enfermeiro \\
\hline $\begin{array}{l}\text { Observar e anotar débito urinário e suas características. Comunicar alterações } \\
\text { ao enfermeiro }\end{array}$
\end{tabular} & \\
\hline $\begin{array}{l}\text { Realizar compressas frias se temperatura > 37,4C e comunicar enfermeiro } \\
\text { enfermeiro }\end{array}$ & \\
\hline Proporcionar aquecimento externo se temperatura < 36 ${ }^{\circ} \mathrm{C}$ e comunicar & \\
\hline
\end{tabular}

